\title{
AN INEQUALITY INVOLVING BESSEL FUNCTIONS OF ARGUMENT NEARLY EQUAL TO THEIR ORDER
}

KEEVE M. SIEGEL

Introduction. Watson $[1 ; 2]$ derives certain inequalities involving Bessel functions of nearly equal argument and order, namely:

$$
\begin{aligned}
& J_{\nu}(\nu x) \leqq \frac{e^{-\nu P(0, x)}}{\left(1-x^{2}\right)^{1 / 4}(2 \pi \nu)^{1 / 2}}, \\
& J_{\nu}^{\prime}(\nu x) \leqq \frac{e^{-\nu F(0, x)}\left(1+x^{2}\right)^{1 / 4}}{x(2 \pi \nu)^{1 / 2}},
\end{aligned}
$$

where $0<x \leqq 1, \nu>0$, and

$$
F(\theta, x)=\log \frac{\theta+\left(\theta^{2}-x^{2} \sin ^{2} \theta\right)^{1 / 2}}{x \sin \theta}-\cot \theta\left(\left(\theta^{2}-x^{2} \sin ^{2} \theta\right)^{1 / 2}\right) .
$$

He remarks [2] after deriving equation (2) that "The absence of the factor $\sqrt[4]{1-x^{2}}$ from the denominator is remarkable."

This paper obtains an inequality for $J_{\nu}(\nu x)$ where the factor $\left(1-x^{2}\right)^{1 / 4}$ does not appear. The region where it is a better upper bound than (1) is determined.

The notation used is exactly that of Watson [2] and most of the derivation is exactly the same as used by Watson to derive (1).

Derivation.

$$
J_{\nu}(\nu x)=\frac{1}{\pi} \int_{0}^{\pi} e^{-\nu F(\theta, x)} d \theta
$$

Watson shows that $F(\theta, x)$ has the following property

$$
F(\theta, x) \geqq F(0, x)+\frac{1}{2}\left(\theta^{2}-x^{2} \sin ^{2} \theta\right)\left(1+x^{2}\right)^{-1 / 2} .
$$

Since $1 \geqq x>0$ and $\theta^{2}>\sin ^{2} \theta$, then (5) can be replaced by

$$
F(\theta, x) \geqq F(0, x)
$$

or

$$
F(\theta, x)>F(0, x) \quad \text { if } \theta \neq 0 .
$$

Presented to the Society, April 25, 1953, under the title Bessel functions of nearly equal argument and order, by K. M. Siegel and F. B. Sleator; received by the editors April 13, 1953. 
Combining (6) and (4)

(7)

$$
J_{\nu}(\nu x)<\frac{1}{\pi} \int_{0}^{\pi} e^{-\nu P(0, x)} d \theta,
$$

(8)

$$
J_{\nu}(\nu x)<e^{-\nu P(0, x)} \text {. }
$$

(8) is a better upper bound than (1) when $1>\left(1-x^{2}\right)^{1 / 4}(2 \pi \nu)^{1 / 2}$. Since

$$
F(0, x)=\log \frac{1+\left(1-x^{2}\right)^{1 / 2}}{x}-\left(1-x^{2}\right)^{1 / 2}
$$

and

$$
F(0,1)=0,
$$

then as $x \rightarrow 1$ the right side of (1) increases without limit while the right side of (8) approaches 1.

Similar inequalities can be derived for the case when the argument is greater than the order or when Hankel functions are considered instead of Bessel functions.

\section{REFERENCES}

1. G. N. Watson, Bessel functions and Kapteyn series, Proc. London Math. Soc. (2) vol. 16 (1917) pp. 150-174.

2. - A treatise on the theory of Bessel functions, 2d ed., Cambridge, 1948, pp. 252-255.

UNIVERSITY OF MICHIGAN 